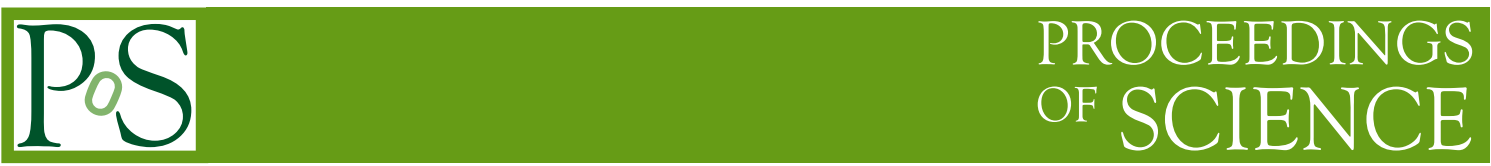

\title{
The hunt for the neutrino hierarchy
}

\section{Martina Gerbino*†}

The Oskar Klein Centre for Cosmoparticle Physics, Department of Physics, Stockholm University, AlbaNova, SE-106 91 Stockholm, Sweden

E-mail: martina.gerbinoefysik.su.se

\begin{abstract}
A novel approach to derive constraints from cosmological datasets on the total neutrino mass $M_{V}$ while taking into account our ignorance of the neutrino mass ordering, either normal $(\mathrm{NH})$ or inverted (IH) is presented. This novel approach is carried out in the framework of hierarchical problems in Bayesian analysis. In this context, the choice of the neutrino mass ordering is modeled via the discrete hyperparameter $h_{\text {type }}$, which we introduce in the usual Markov chain analysis. The preference for either the NH or the IH scenarios is then simply encoded in the posterior distribution of $h_{\text {type }}$ itself. Current cosmic microwave background (CMB) and baryon acoustic oscillation (BAO) measurements show a weak preference for the NH scenario, with odds of 3:2. Concerning next-generation cosmological experiments, forecasts suggest that the combination of upcoming $\mathrm{CMB}$ (COrE) and BAO surveys (DESI) may determine the neutrino mass hierarchy at a high statistical significance if the mass is very close to the minimal value allowed by oscillation experiments (9:1 preference of $\mathrm{NH}$ versus $\mathrm{IH}$ for $\mathrm{NH}$ and a fiducial value of $M_{v}=0.06 \mathrm{eV}$ ). On the contrary, if the sum of the masses is of the order of $0.1 \mathrm{eV}$ or larger, even future cosmological observations will be inconclusive. The unbiased limit on $M_{v}$ we obtain is crucial for ongoing and planned neutrinoless double beta decay searches.
\end{abstract}

Neutrino Oscillation Workshop

4 - 11 September, 2016

Otranto (Lecce, Italy)

\footnotetext{
* Speaker.

${ }^{\dagger}$ Based on work in collaboration with Massimiliano Lattanzi, Olga Mena and Katherine Freese [1].
} 


\section{Introduction}

According to the standard theory of neutrino oscillations, the observed neutrino flavours $v_{\alpha}$ $(\alpha=e, \mu, \tau)$ are a superposition of the massive eigenstates $v_{i}(i=1,2,3)$. From neutrino oscillation data, we know that at least two out of the three mass eigenstates should be massive, as the solar $\Delta m_{21}^{2}$ and atmospheric $\left|\Delta m_{31}^{2}\right|$ mass splittings are measured with percent accuracy by current experiments [2]. Depending on the sign of $\Delta m_{31}^{2}$, two possible mass orderings can be identified: the normal hierarchy (NH hereafter), in which $m_{1}<m_{2}<m_{3}$, and the inverted hierarchy (IH in what follows), in which $m_{3}<m_{1}<m_{2}$. Assuming that the mass of the lightest eigenstate is zero, it is possible to obtain a lower bound on the sum of neutrino masses of $M_{v} \simeq 0.06 \mathrm{eV}$ in NH $\left(M_{V} \simeq 0.1 \mathrm{eV}\right.$ in $\left.\mathrm{IH}\right)$ from neutrino oscillation measurements. Neutrino mixing phenomena are not sensitive to the individual neutrino masses nor to the overall mass scale. Consequently, in the absence of a robust measurement of the neutrino mass ordering, a desirable bound on the sum of the neutrino masses $M_{v}$ would be one which relies on the less informative possible assumption about the hierarchical distribution of the total mass among the three eigenstates. This is a typical example of a hierarchical model in statistical inference, where the distribution of the parameters of the model under scrutiny (e.g. $M_{V}$ ) conditionally depends on the so-called hyperparameters (e.g. the hierarchy). We therefore propose a novel method to get a hierarchy-independent bound on $M_{v}$, by means of the discrete hyperparameter $h_{\text {type }}$ (that can in practice be identified with the sign of the atmospheric mass splitting), introduced in the standard Monte Carlo Markov chain (MCMC) analysis. This innovative strategy benefits from the fact that the sensitivity to the neutrino hierarchy is simply encoded in the posterior probability distribution of $h_{\text {type }}$. Cosmology provides one of the most suitable places where to test and extract the neutrino mass ordering [3, 4]. Apart from cosmological probes, there also exist laboratory avenues which are sensitive to the absolute mass scale, such as the neutrinoless double $\beta$ decay $(0 v 2 \beta)$ searches [5]. Planned $0 v 2 \beta$ experiments might have the required sensitivity to completely cover the region of the parameter space where a positive signal is expected in the case of IH distribution of the total neutrino mass. Robust limits on the total neutrino mass coming from cosmology can further reduce the allowed region of the parameter space where to look for $0 v 2 \beta$ events.

\section{Method, data and results}

In this work, the model parameters are extracted conditionally on the choice of the neutrino mass hierarchy. This choice is modelled by introducing a discrete hyperparameter $h_{\text {type }}$ that can take two values, corresponding to $\mathrm{NH}$ and $\mathrm{IH}$ (i.e to $\operatorname{sign}\left(\Delta m_{31}^{2}\right)=+1$ or -1 , respectively). Since little is known from current experiments about the preference for one of the two neutrino hierarchies, we assign equal a priori probability to the two possible outcomes that $h_{\text {type }}$ could take. We therefore perform a MCMC analysis of an eight-dimensional parameter space: the usual set of six cosmological parameters in the $\Lambda \mathrm{CDM}$ scenario; the lightest eigenstate mass which is equivalent to $m_{1}\left(m_{3}\right)$ in the $\mathrm{NH}(\mathrm{IH})$ scenario (the mass of the remaining two neutrino states is set through the solar and atmospheric mass gaps as measured by oscillation experiments); the discrete hyperparameter $h_{\text {type }}$. At each step, we extract $h_{\text {type }}$ from $\{\mathrm{NH}, \mathrm{IH}\}$ and therefore handle our ignorance about the true hierarchical distribution of the mass as a nuisance parameter. In addition, the pos- 
terior distribution of $h_{\text {type }}$ for a given datasets contains information about the preference for one of the two hierarchies from that dataset. This information is conveyed by reporting the "odds" for NH vs. IH, i.e. the ratio between the marginalized probabilities for the two hierarchies.

We employ measurements of CMB anisotropies in temperature and polarisation from the Planck mission [6] (labeled as Planck TT+lowP and Planck TT,TE,EE+lowP depending on whether small-scale polarization measurements are employed), as well as geometrical information from galaxy clustering, i.e. via the $\mathrm{BAO}$ signature, making use of the BAO results from the $6 \mathrm{dF}$ Galaxy Survey (6dFGS) [7] and from the BOSS DR11 LOWZ and CMASS samples [8]. We also perform forecasts for future CMB and galaxy clustering data. Concerning CMB measurements, we consider a future COrE-like [9] satellite mission. Future galaxy clustering data are added by means of the expected observations of the BAO signal along and across the line of sight from the Dark Energy Instrument (DESI) Experiment [10].

Concerning current datasets, CMB data alone are not sensitive to the different mass parameterizations. This is due to the broad bound that the CMB data set on the total neutrino mass. In fact, the potential for cosmological observations to discriminate between the two mass orderings mainly relies on the capability to push the upper limit on $M_{V}$ below $0.1 \mathrm{eV}$, the minimal value of the mass allowed by oscillation data in the case of IH. The region of masses with $M_{v} \simeq 0.1 \mathrm{eV}$ is the one where the mass patterns predicted by $\mathrm{NH}$ and $\mathrm{IH}$, and the resulting cosmological perturbations, differ the most. The differences are however below the sensitivity of present, and possibly also future, experiments, and the dominant contribution to the constraining power still comes from the amount of volume in parameter space available to the two models. The inclusion of BAO measurements results in much tighter neutrino mass bounds. The combination of the cosmological data then starts to be sensitive to the region of in which the $\mathrm{NH}$ and the IH scenarios correspond to different neutrino mass spectra. To conclude, the combination of CMB and BAO slightly favors the NH scheme (4:3 odds in favor of $\mathrm{NH}$, without using CMB small-scale polarization, or 3:2 if we use it). Concerning the sensitivity of future cosmological measurements, we have considered three possible fiducial scenarios: two NH schemes, one with $M_{v}=0.06 \mathrm{eV}$ and the other one with $M_{v}=0.1 \mathrm{eV}$, and one IH scenario, also with $M_{v}=0.1 \mathrm{eV}$. In the case of the fiducial model with $M_{v}=0.06 \mathrm{eV}$, we find a 9:1 preference of NH versus IH. On the contrary, for the $M_{v}=0.1 \mathrm{eV}$ case, the data still remain completely uninformative for what concerns the mass splitting. This confirms the fact that the capability of future cosmological observations to discriminate the hierarchy mainly relies on the possibility of excluding $M_{v} \geq 0.1 \mathrm{eV}$ with a high statistical significance, as in the case for the fiducial model with $M_{v} \geq 0.06 \mathrm{eV}$. When instead $M_{v}=0.1 \mathrm{eV}$ (or larger), as in the other two fiducial models, the two mass orderings should be disentangled through the effect of the individual neutrino masses on the evolution of cosmological perturbations. Our findings clearly indicate that this is beyond the reach of next-generation $\mathrm{CMB}$ and $\mathrm{BAO}$ experiments.

\section{Discussion and conclusions}

We shall now discuss the implications of our analyses for $0 v 2 \beta$ searches. In analogy to [11], we extend the analysis discussed previously, extracting the posterior distribution for the Majorana mass $m_{\beta \beta}$, i.e. the neutrino mass parameter constrained by $0 v 2 \beta$ experiments [12]. We illustrate in the upper panel of Fig. 1 the $68 \%$ and $95 \%$ CL probability contours in the $M_{v}-m_{\beta \beta}$ plane from 
a combination of current data. We also depict together the tightest bounds on neutrinoless double beta decay searches, coming from the KamLAND-Zen experiment [13] . There exist two equally allowed possible regions for $m_{\beta \beta}$, due to the existing neutrino mass hierarchy ambiguity. Interestingly, current sensitivities to $m_{\beta \beta}$ start to reach the allowed region by cosmological and oscillation measurements, and, consequently, if nature has chosen the inverted hierarchy, a positive signal from neutrinoless double beta decay searches could be imminent (providing of course neutrinos possess a Majorana character and barring exotic physical scenarios). Concerning forecasted results, we
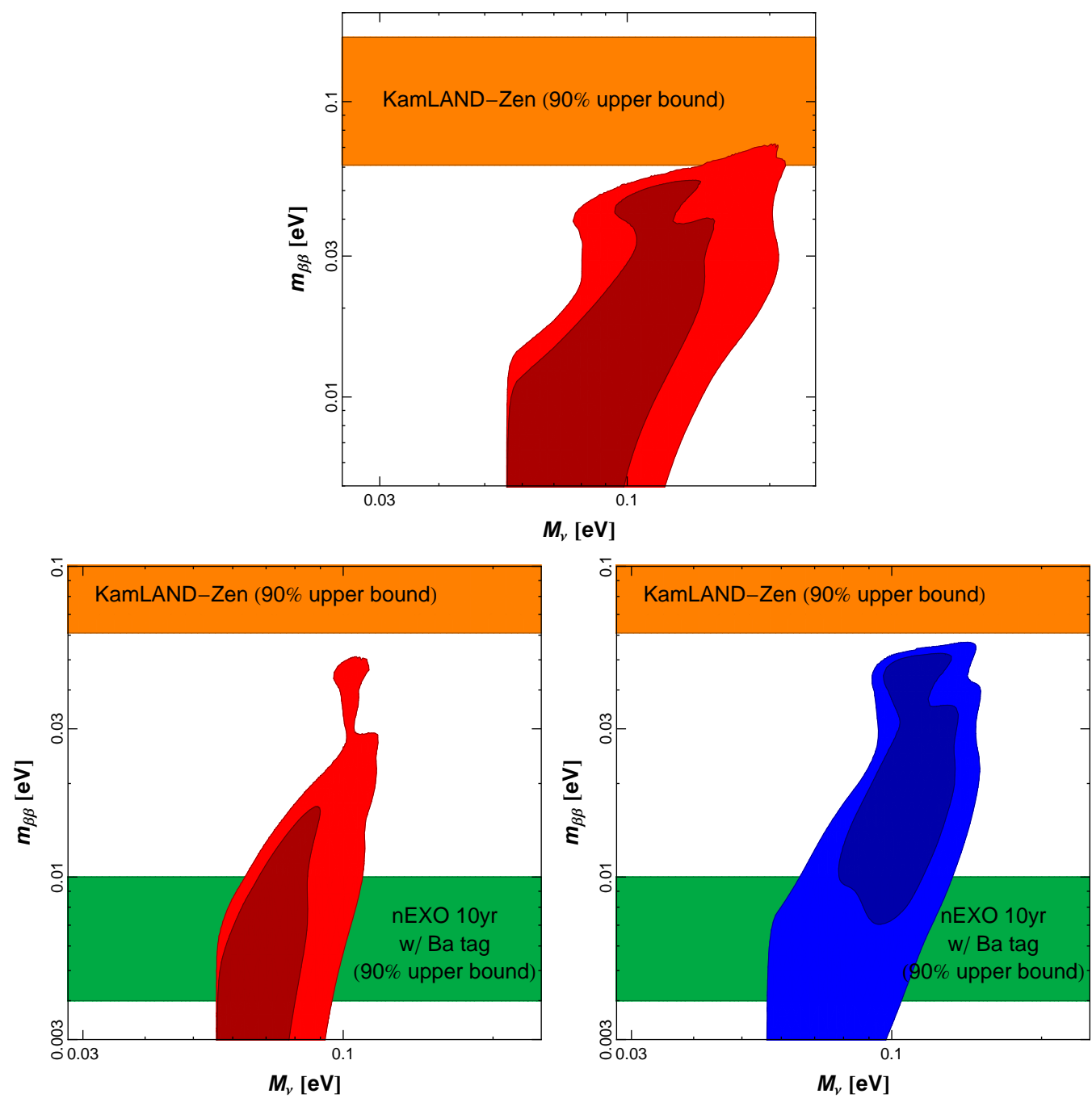

Figure 1: Two-dimensional probability contours in the $M_{v}-m_{\beta \beta}$ plane for the Planck TT,TE,EE+lowP+BAO combination (upper panel), and for COrE+DESI combination and a fiducial value of $M_{V}=0.06 \mathrm{eV}$ (bottom left panel) and $M_{V}=0.1 \mathrm{eV}$ (bottom right panel). The horizontal bands correspond to the range of $90 \%$ CL upper bound on the Majorana mass from the KamLAND-Zen experiment (orange) and from a nEXO-like experiment (green). Adapted from [1].

consider two fiducial models with NH mass ordering and either $M_{v}=0.06 \mathrm{eV}$ or $M_{v}=0.1 \mathrm{eV}$. The bottom panels of Fig. 1 depict the two-dimensional contours in the $M_{v}-m_{\beta \beta}$ plane for the 
two possible fiducial models mentioned above, along with the expected upper bounds from a future, nEXO-like [14] neutrinoless double beta decay experiment. In the case of $M_{v}=0.1 \mathrm{eV}$ the prospects of observing a positive signal from a future $0 v 2 \beta$ decay are very good (provided neutrinos are Majorana and the mass mechanism is responsible for $0 v 2 \beta$ decay). In this situation the hierarchy could be extracted by neutrinoless double beta decay itself, since a positive signal characterized by $m_{\beta \beta} \simeq 0.05 \mathrm{eV}$ would suggest an $\mathrm{IH}$ scenario. Alternatively, a positive signal characterized by $m_{\beta \beta} \simeq 0.02 \mathrm{eV}$ in combination with the expected sensitivity of $\sigma\left(m_{\beta \beta}\right) \sim 0.01 \mathrm{eV}$ would point to a $\mathrm{NH}$ scenario and discard the IH scenario with high statistical significance. If, on the other hand, $M_{v}$ is closer to the minimal value allowed in the NH scenario, the sensitivity of future neutrinoless double beta decay searches may not be enough, due to the possible disruptive interference played by oscillation parameters in the definition of the Majorana mass. The good news is that, in this case, cosmology will be able to tell us about the neutrino mass hierarchy with compelling statistical significance.

\section{References}

[1] M. Gerbino, M. Lattanzi, O. Mena and K. Freese, arXiv:1611.07847 [astro-ph.CO].

[2] C. Patrignani et al. [Particle Data Group], Chin. Phys. C, 40, 100001 (2016).

[3] J. Lesgourgues, S. Pastor and L. Perotto, Phys. Rev. D 70, 045016 (2004) [hep-ph/0403296].

[4] S. Hannestad and T. Schwetz, JCAP 1611, no. 11, 035 (2016) [arXiv:1606.04691 [astro-ph.CO]].

[5] O. Cremonesi and M. Pavan, Adv. High Energy Phys. 2014, 951432 (2014) [arXiv:1310.4692 [physics.ins-det]].

[6] N. Aghanim et al. [Planck Collaboration], Astron. Astrophys. 594, A11 (2016) [arXiv:1507.02704 [astro-ph.CO]].

[7] F. Beutler et al., Mon. Not. Roy. Astron. Soc. 416, 3017 (2011) [arXiv:1106.3366 [astro-ph.CO]].

[8] L. Anderson et al. [BOSS Collaboration], Mon. Not. Roy. Astron. Soc. 441, no. 1, 24 (2014) [arXiv:1312.4877 [astro-ph.CO]].

[9] F. R. Bouchet et al. [COrE Collaboration], arXiv:1102.2181 [astro-ph.CO].

[10] M. Levi et al. [DESI Collaboration], arXiv:1308.0847 [astro-ph.CO].

[11] M. Gerbino, M. Lattanzi and A. Melchiorri, Phys. Rev. D 93, no. 3, 033001 (2016) [arXiv:1507.08614 [hep-ph]].

[12] J. Schechter and J. W. F. Valle, Phys. Rev. D 25, 2951 (1982).

[13] A. Gando et al. [KamLAND-Zen Collaboration], Phys. Rev. Lett. 117, no. 8, 082503 (2016) Addendum: [Phys. Rev. Lett. 117, no. 10, 109903 (2016)] [arXiv:1605.02889 [hep-ex]].

[14] https://www-project.slac.stanford.edu/exo/ 